\title{
Transcranial direct current stimulation enhances recovery from motor deficits following hypoxia-ischemia in neonatal
} rats

\author{
Christopher L. Anderson ${ }^{1,2 *}$, Benjamin J. Tessler', Thomas B. DeMarse², Martha Douglas-Escobar ${ }^{3}$, Candace Rossignol ${ }^{4}$, Rachel Nelson ${ }^{5}$, Aditya \\ K. Kasinadhuni ${ }^{1}$, Michael King ${ }^{5}$, Prodip Bose ${ }^{5}$, Michael D. Weiss ${ }^{4}$ and Paul R. Carney ${ }^{2}$ \\ 1J. Crayton Pruitt Family Department of Biomedical Engineering, University of Florida, Gainesville, Florida, USA \\ ${ }^{2}$ Department of Neurology, University of North Carolina, Chapel Hill, North Carolina, USA \\ ${ }^{3}$ Department of Pediatrics, University of California San Francisco, San Francisco, California, USA \\ ${ }^{4}$ Department of Pediatrics Division of Neonatology, University of Florida, Gainesville, Florida, USA \\ 5Brain Rehabilitation Research Center, North Florida/South Georgia VA Health System, Gainesville, Florida, US
}

\begin{abstract}
Prenatal cerebrovascular stroke can cause permanent damage to the brain followed by deficits in neuro-muscular functioning. We hypothesize that recovery can be enhanced by transcranial direct current stimulation (tDCS) through changes in cortical plasticity. Unilateral hypoxia-ischemia (HI) was produced in rats on postnatal day 7 (P7). At P21, anodal tDCS was given to HI pups for 7 days. tDCS treated HI pups showed improved weight, grip strength, gait, motor function, and concentrations of brain-derived neurotropic factor cortex ratios compared to non-treated HI animal controls. These findings support tDCS following HI as an effective therapeutic for neonatal stroke.
\end{abstract}

\section{Introduction}

Stroke is defined as permanent brain damage due to obstruction of blood or oxygen to the brain. It is known that neonatal strokes are recognized in about 1 of 4000 live births per year [1]. Strokes that have occurred in newborns often result in life long impairments including reduced or lost control of sensory and motor functions, aphasia, seizures, learning impairments, cerebral palsy, and death. To date, the most promising rehabilitative treatment is physical therapy (PT) [2]. However, despite early PT, more a significant percentage will progress to severe motor disability that will affect their basic activities of daily living [2]. Hence, there is need for more effective rehabilitative therapies, particularly for targeted therapies that prevent the secondary and tertiary damage processes associated with severe stroke.

Over the last decade there has been a resurgence of interest in studying the potential therapeutic effects of non-invasive brain stimulation [3-5] and its potential benefits of cognitive and memory enhancement [6,7], and neurological disease [8,9] including schizophrenia [6] Parkinson disease [10], and stroke [11,12]. Noninvasive brain stimulation includes a number of technologies including transcranial magnetic stimulation (TMS) and transcranial direct current stimulation (tDCS), among others. In tDCS, surface electrodes are placed on the scalp and a weak direct current (anodal or cathodal) is applied. The current generates a weak electric field that alters the resting membrane potential of neurons within that field [13]. tDCS is considered a safe and unobtrusive way, in children and adults [14], to alter regional cerebral processes and elicit changes in physical and cognitive functioning, with the added benefit that it does not require any surgical procedures, unlike other forms of neurostimulation such as deep brain stimulation (DBS). There are now a number of studies that have shown that anodal tDCS of the lesioned motor region following stroke can result in a significant improvement of motor function in adult humans compared to sham stimulation control groups [12].

Plasticity in children's brains during development is elevated relative to adults [15]. We hypothesize that motor recovery is facilitated by employing tDCS to further enhance natural plasticity during motor reorganization of the infant's brain following hypoxic-ischemia (HI). However, before testing the effects of $\mathrm{tDCS}$ on the motor behavior of human infants with neonatal $\mathrm{HI}$, it is important to perform preclinical tests on a comparable rat model to determine safety, efficacy, and natural mechanisms involved. In this study, we use a well-established Rice-Vannucci model [16] in neonatal HI rat pups in which tDCS is applied to determine whether tDCS will enhance motor performance relative to non-HI controls.

Using a ring electrode, animals were electrically stimulated with an anodal current protocol of short interstimulation intervals at $200 \mu \mathrm{A}$ each day for one week that were paralleled by multiple neurofunctional tests including negative geotaxis, grip strength, and gait analysis. Following stimulation and behavior tests, tissue and protein samples were collected and used to measure brain-derived neurotropic factor (BDNF) levels, which is a marker for plasticity, in a small sample group

Correspondence to: Christopher L. Anderson, Department of Biomedical Engineering, University of Florida, 1275 Center Drive, Biomedical Sciences Building JG-56 Gainesville, FL 32611-6131, USA, E-mail: cla7390@ufl.edu

Received: May 29, 2017; Accepted: June 16, 2017; Published: June 19, 2017 
of stimulated and non-stimulated animals. We show that tDCS has a neurophysiological effect on the neonatal rat brain after $\mathrm{HI}$ insult, with promising outcomes showing improvement in motor function after stimulation.

\section{Results}

To assess in vivo the degree of $\mathrm{HI}$ injury, six high-field magnetic resonance (MR) images obtained from a single rat pup (P15) under anesthesia eight days after $\mathrm{HI}$ injury were obtained (Figure 1) (Agilent Magnex Scientific 11.1T $40 \mathrm{~cm}$ horizontal magnet at the University of Florida's McKnight Brain Institute). The cortical damage including necrosis and white matter injury in the right hemisphere is clearly visible in this animal.

The individual weight of each animal was recorded each day, starting on the day of HI surgery and ending one week following stimulation (Figure 2a). At P23 the HI injured group of rat pups that weren't stimulated $(n=13)$ weighed an average of $46.00 \pm 3.89 \mathrm{~g}$, the HI group that were to be stimulated $(n=14)$ weighed $45.43 \pm 3.82 \mathrm{~g}$, and the sham group that wasn't stimulated or injured $(n=8)$ weighed $56.13 \pm$ $2.75 \mathrm{~g}$. Over the course of the following week, the weights of stimulated animals increased significantly relative to non-stimulated $\mathrm{HI}$ animals $(\mathrm{p}<0.01)$. At the end of tDCS treatment, animals weighed $105.86 \pm$ $4.61 \mathrm{~g}$ and reached the weights of the sham (untreated) controls (110.88 $\pm 6.44 \mathrm{~g}$ ). The HI animals weighed less than both the sham controls and the HI-tDCS treated animals $94.77 \pm 4.73 \mathrm{~g}$.

Negative geotaxis is a righting reflex in which pups naturally turn to their heads upward after they are placed facing downward on an incline plane. To successfully perform the test pups, require the ability to pivot themselves about a front paw. The time it takes for an animal to right itself and complete this task is measured. All pups underwent baseline testing for 2 days prior to stimulation to establish baseline estimates, with average times collected each day (Figure 2b). On the first day of the stimulation period, HI and HI-tDCS treated animals required significantly more time to right themselves relative to the sham control

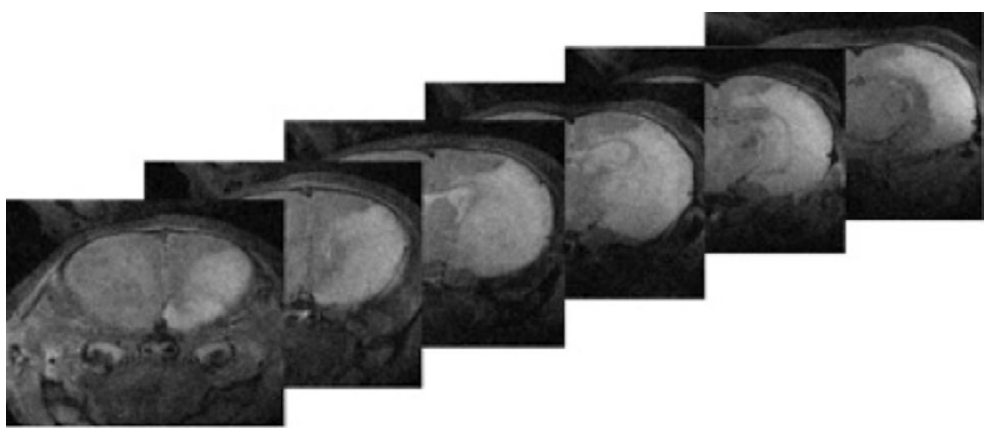

Figure 1. 11T T2 In vivo Magnetic Resonance Imaging of a P15 rat 8 days after Hypoxic-Ischemic injury. Cortical damage can be seen in the cortex, subcortical white matters, and hippocampus, represented by the white regions. This corresponds to the HI injury.
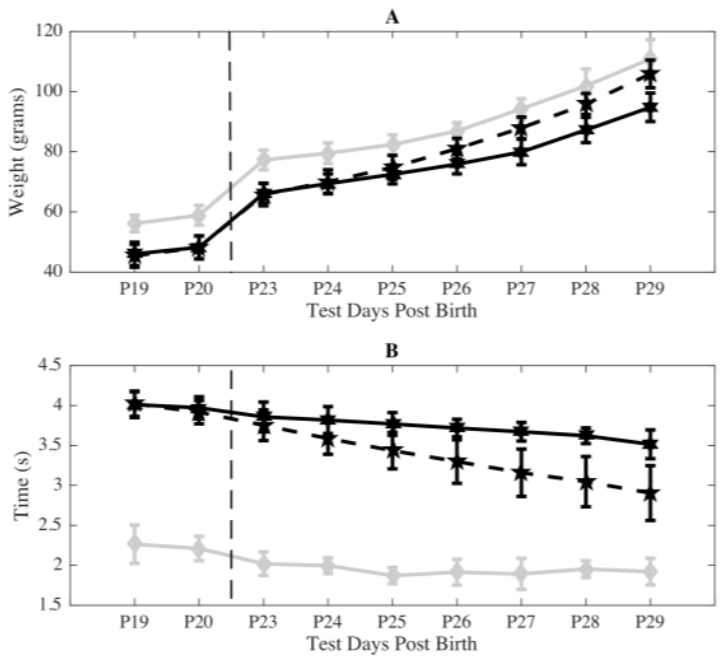
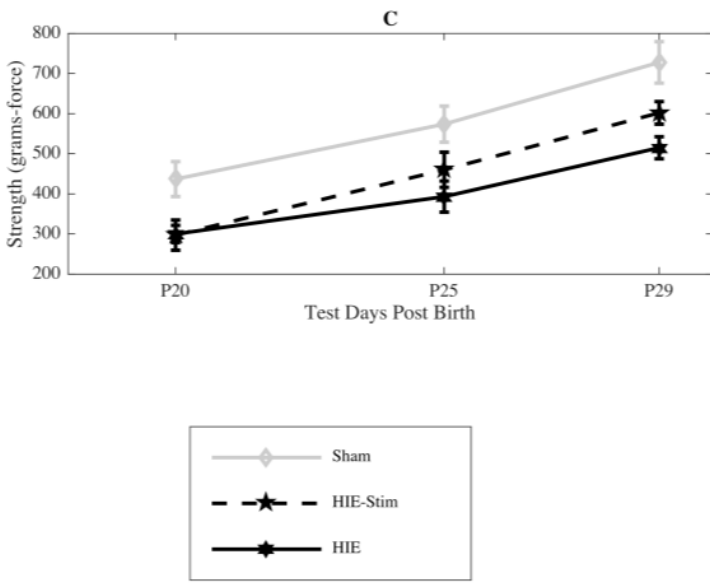

Figure 2. (a) Weight was examined prior to and after treatment. Prior to treatment there was no difference between the HI group (black, $n=13$ ) and the HI-tDCS group (dashed, $n=14$ ). After stimulation, the HI-tDCS group had a marked increase in weight gain, which differed significantly from the HI group at P26 (*p<0.01) with no significant difference between the HI-tDCS and sham group (gray, $\mathrm{n}=8$ ) at this time point.

(b) Negative Geotaxis: The pups in both the HI (black, n=13) and HI-tDCS (dashed, n=14) groups were similar before testing with a marked increase in the time needed to rotate upward when compared with sham pups (gray, $n=8$ ). After stimulation, there was a marked improvement in the negative geotaxic reflex in the HI-tDCS group compared with the HI group $(* \mathrm{p}<0.01$ at P25 and continuing for all other time points).

(c) Grip Strength: Grip strength was tested on the contralateral paw to the cortical injury. The Sham group (gray, $\mathrm{n}=8$ ) had higher baseline grip strength and there was no difference in the HI groups prior to testing. The HI-tDCS group (dashed, $\mathrm{n}=14$ ) had an increase in strength compared with the HI group (black, $\mathrm{n}=13$ ) after 4 days of treatment $(* \mathrm{p}<0.01$ ). The HI-tDCS group approached the Sham controlled group at P29. 
group $(\mathrm{p}<0.01)$. Following the seven-day stimulation period, the tDCS treated $\mathrm{HI}$ animals righted themselves at an average time of $2.91 \pm 0.34$ $\mathrm{s}$ compared to the non-treated $\mathrm{HI}$ animals that averaged a time of 3.52 $\pm 0.18 \mathrm{~s}(\mathrm{p}<0.01)$. Both groups were slower than the sham control group, $1.92 \pm 0.17 \mathrm{~s}(\mathrm{p}<0.01)$.

Grip strength is a common measure of the rat's neuromuscular system following HI. Grip strength was tested on the contralateral paw to the cortical injury. Data from this test was collected in each group before, during, and on the last day of the treatment period and means plotted in Figure 2c. Before treatment, grip strength was equivalent in HI and HI-tDCS treated animals and each were weaker than sham animals that had not been injured $(\mathrm{p}<0.01)$. At P25, midway through treatment, grip strength had increased significantly in the HI-tDCS group compared with the untreated HI animals and this difference continued for the remainder of the measured time points $(\mathrm{p}<0.01)$. At the end of treatment, the HI- treatment tDCS group's cohort grip strength approached the sham controls.

To assess the individual's condition in their ability to walk, gait analysis data was collected at two time points, before (P21) and after (P29) tDCS treatment in two of the animals from each group. Results can be seen in Figure 3. Base of Support, Print Area, and Phase Dispersion Area measurements were all significantly different $(\mathrm{p}<$ .05 ) for various models using the normal theory linear mixed model framework (two-way ANOVA with subject-specific random effects) with variables of group and time point. The Print Area data was shown to be significantly different $(\mathrm{p}<0.05)$ when using the model comparing group and time point additive vs time point, comparing time point vs intercept, and comparing group and time point interaction vs intercept. Base of Support was shown to be significantly different $(\mathrm{p}<0.05)$ when using the model comparing group and time point interaction vs group and time point additive, and comparing group and time point interaction vs intercept. Phase Dispersion Area from right foot to left hand was shown to be significantly different $(p<0.05)$ when using the model comparing group and time point interaction vs group and time point additive. Phase Dispersion Area from left foot to right hand was shown to be significantly different $(\mathrm{p}<0.05)$ when using the model comparing group and time point additive vs time point, and comparing group vs intercept.

The ratio of brain-derived neurotrophic factor (BDNF) expression in injured versus non-injured cortex for each rat pup is shown in Figure 4. BDNF expression was higher in tDCS treated animals compared to non-treated HI control animals when tested in a subset of animals on P30 $(n=5$ for HI-tDCS, $n=4$ for HI) and P36 $(n=2$ for HI-tDCS, $n=1$ for $\mathrm{HI}$ ). The BDNF ratio increased by an average of 1.202 from tDCS treated animals to non-treated HI control animals at P30 and 2.602 at P36.

\section{Discussion}

From this study, we can conclude that chronic tDCS treatment is innocuous and improves motor deficits associated with HI injury, with the tDCS treated rat pups showing improvement in overall movement ability, strength, and weight gain. Furthermore, results suggest that improvement in motor function most likely represent augmentation of plasticity, which can be seen in neonates and is supported by the BDNF results. The results of this study are significant in that, unlike other studies that aim to assist in preventing functional loss as a result of injury, an improvement was seen in tDCS treated animals during the tertiary or chronic phase of the injury. Prior to stimulation, the injured animals showed significant motor deficits and changes in behavior compared to the sham controls. While aiming to prevent the $\mathrm{HI}$ event from damaging the neonatal brain and hindering development is crucial, having a treatment for the large population of neonates with established deficits and cortical damage is also important, making the results of this study, showing functional improvement outside of the acute phase of the injury, significant.
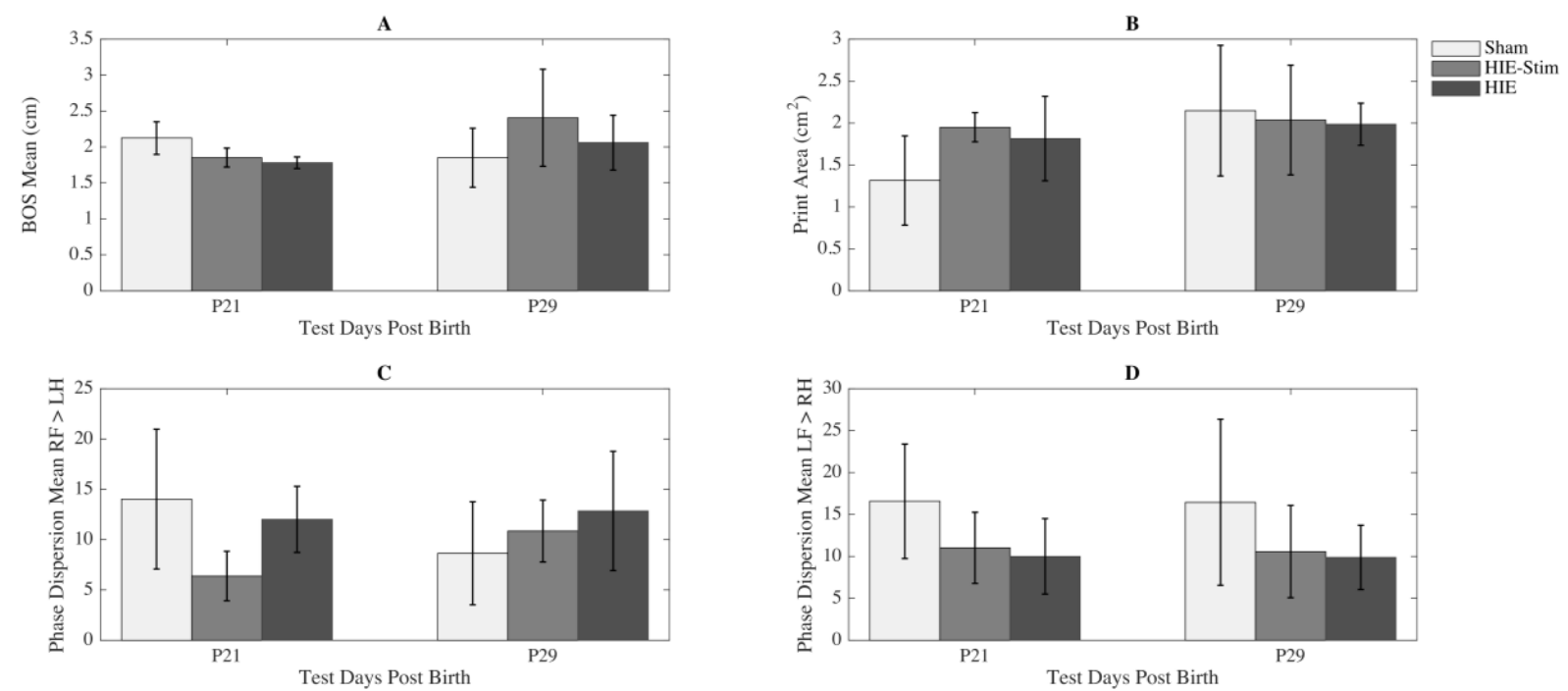

Figure 3. Catwalk Gait Parameters:

(a) The Base of Support is the average width $(\mathrm{cm})$ between the Front Paws of each animal when walking.

(b) The Print Area is the surface area of each Hind Paw when the animal is making contact while walking. Phase Dispersion Area is the mean temporal placement of two paws within two consecutive initial contacts, used to measure the

coordination of the animal's paw placement. Here we looked at the Right Front to Left Hind

(c) and Left Front to Right Hind

(d) phase dispersions for the Sham (gray, $n=2$ ), HI (black, $n=2$ ), and HI-tDCS (dashed, $n=2$ ) groups. 


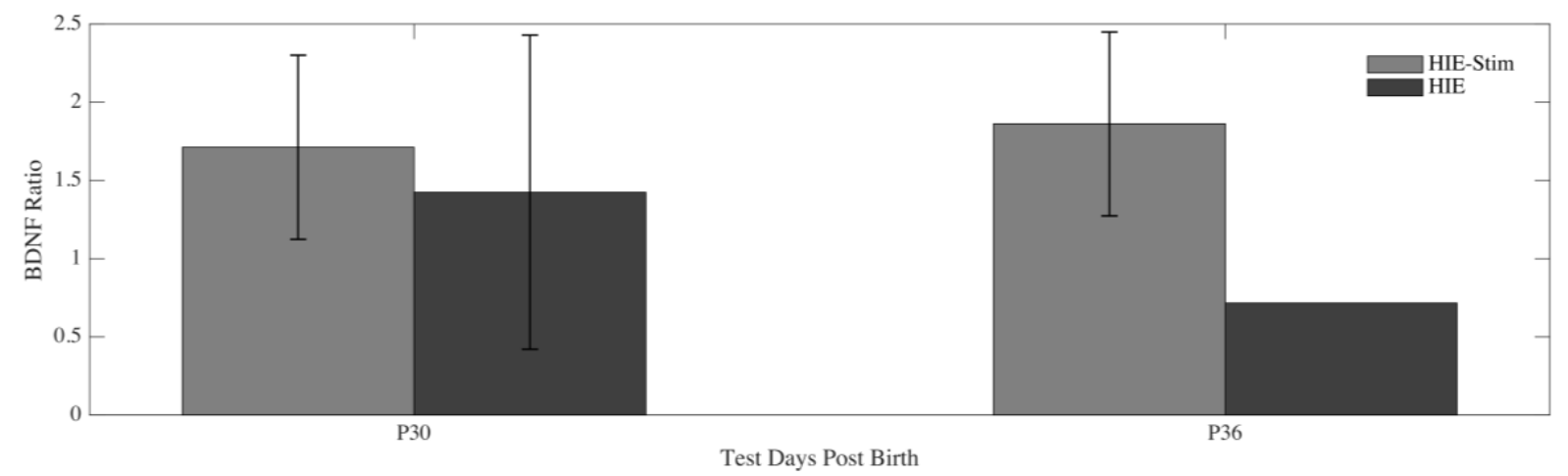

Figure 4. Brain-Derived Neurotropic Factor: The ratio of BDNF from the cortex of the injury side of the rat pup brain to the cortex of the non-injured side. BDNF was calculated from protein samples from both the HI-tDCS (dashed) and HI (black) animal groups. Samples were collected at postnatal days 30 (P30, n=5 for HI-tDCS, n=4 for HI) and P36 (n=2 for HI-tDCS, $\mathrm{n}=1$ for $\mathrm{HI}$ ).

It is believed that tDCS may facilitate the remapping of the brain via cortical plasticity by blocking or suppressing the activation potential of neurons [17]. With $\mathrm{tDCS}$, the plastic nature of the developing brain could be enhanced $[17,18]$ as a putative mechanism for motor recovery. The pups in the HI group improved over time suggesting inherent plasticity over time, While the HI-tDCS treated group demonstrated improvement of motor function much more rapidly over time and were approaching the sham control motor abilities. After tDCS treatment, the HI injured brain hemisphere did show an increase in BDNF levels compared to those that did not receive tDCS and parallels similar findings from other groups with this measure $[5,19]$.

We also observed significant weight gain in the stimulated vs non-stimulated HIE control. This increase would be consistent with improved motor behavior observed in other measures and reflected in improved ability of the life sustaining tasks vise vie improved suck, that are involved with food consumption or an increase in appetite of the stimulated group.

The benefits of an animal study that these experiments bring into consideration comes from the fact that the rats scalp was peeled away for the entire duration of the week of tDCS. There is much debate about the underlying mechanisms behind the effects of tDCS [20]. There has been growing knowledge of how peripheral nerve stimulation can affect the CNS reportedly producing many similar effects to that of tDCS [21]. The data suggesting a high percentage of current shunting through the highly conductive scalp may suggest that the main mechanism of tDCS depends on the nerves under the skin [22]. This has casted much doubt within the public and scientific community [23]. However, in our experiment electrodes were attached directly to the skull and surrounded by dental cement which might greatly reduce any stray current through the scalp.

Location of stimulation may be another significant factor. For example, there may be advantages to stimulating over the noninjured side of the brain, because of intact connections, well known current densities and topography, and less disturbance by the lesion [11]. In this study, we employed anodal neurostimulation. However, examining the role of cathodal stimulation may be equally important, especially in regard to the concept of competition for neural pathways and recreating a balance in activity. The time points of both the injury development and the period in which to stimulate need to be considered when developing the appropriate protocol as well. Tracking behavior results at several times after stimulation will help to determine if tDCS treatment has a lasting improvement on the motor function following HI injury. Most noninvasive brain stimulations studies have targeted the hand area of M1 to improve motor hand function [5]. Our results suggest that diffuse stimulation of M1 motor area may be necessary to promote recovery. However, future studies may employ both focal and non-focal arrangement of electrodes in order to induce more widespread neuromodulation in multiple functional brain areas and systems following hypoxic-ischemia. Moreover, depending on the location and size of the cortical injury, tDCS might not be efficient to improve a post stroke deficit, especially if the perilesional brain tissue does not contribute to the brain function that is impaired. Task free functional MR imaging might be used to clarify whether the perilesional functional activation pattern can predict the individual response to tDCS. However, even if the cortical area that is targeted by tDCS is relatively far away from the site of cortical infarction, it is likely that electrical current will spread to all areas of the brain due to the distribution of the fluid space filled with cerebrospinal fluid. Modeling the expected field distribution of the tDCS-induced tissue current in the brain may provide estimates as to which brain regions are effectively stimulated by tDCS based on the calculated field distribution [24].

\section{Methods}

\section{Animal Model of Hypoxia-Ischemia (HI)}

All protocols were approved by the University of Florida's Institutional Animal Care and Use Committee. Sprague-Dawley rat pups were ordered from Harlan Labs (now Envigo). For this study, the Rice-Vannucci model for neonatal HI $[25,26]$ was used. This widely used surgical technique creates relatively consistent neonatal hypoxicischemic models [27]. Rat pups were removed from their den at P7 and a random subset of those pups from each litter were individually anesthetized with isoflurane [3\%]. The anesthetized pups were then placed on a $37^{\circ} \mathrm{C}$ heating pad and anesthesia was provided through a small plastic tube, attached to the nose and mouth. A $1 \mathrm{~cm}$ midline incision was made longitudinal in the neck and the left common carotid artery (CCA) was found and then ligated with a cauterizing tool. The incision was closed using a cyanoacrylate adhesive and then the pup was placed back in the dams for 90 minutes to recover. The average time for each ligation was 8 minutes. For the sham control group, the remaining pups from each litter underwent anesthesia and had the same incision made on the neck but was then sealed without any manipulation of the CCA.

Pups were then placed in a hypoxic chamber and exposed to either a mixture of $8 \%$ oxygen and $92 \%$ nitrogen gas for 150 minutes or a 
normal oxygen atmosphere (Sham control). Temperature within the chamber was maintained at $37^{\circ} \mathrm{C}$ using a heating pad. At the end of the 150 minutes, pups were returned to the dam and there until weaning from the mother at P21.

\section{Magnetic resonance imaging}

At P15 (8 days after HI injury), animals where anesthetized with isoflurane (2\%) and scanned with an 11T MRI system (Agilent Magnex Scientific $11.1 \mathrm{~T} 40 \mathrm{~cm}$ horizontal magnet at the University of Florida's McKnight Brain Institute). The head of the rat pup was positioned in a custom-made cradle with an adjusted bite-bar to maintain head position. Anesthesia, respiration, and body temperature were monitored throughout the scanning process. T2-weighted 11 Tesla scans were acquired with $0.5-\mathrm{mm}$ slices to assess the cortical damage throughout the brain because of the HI injury.

\section{Transcranial direct current stimulation}

After weaning at P21 (14 days' post injury) both the HI and sham rat pups from each litter were removed from the dams and individually anesthetized with isoflurane (4\%). Most clinical tDCS applications use rectangular-pad electrodes or a configuration of smaller ring electrodes. Work by Datta (2009) has revealed that ring electrodes enhance focality and have a more targeted brain modulation [28]. For this reason, and to avoid shunting that is involved in the larger surface electrodes, we also used a ring electrode in this study (Figure 5a). To ensure consistent placement of the stimulating electrode throughout the stimulation period the scalp was incised and a metal stimulating electrode was placed over the skull and cemented into place and illustrated in Figure 1. A small incision was made over the rat pup's cranium, exposing approximately $1 \mathrm{~cm} 2$ of the skull. A $9 \mathrm{~mm} 2$ ring electrode which served as anode was then placed onto the area of the cranium roughly corresponding to area of the ischemic injury and cemented into place using a glass Ionomer luting dental cement (Prevest Denpro Poly Zinc Dental Cement) onto the right frontal epidural space. This cement was chosen due to its non-toxic components and did not require the use of cranial screws, maintaining the ideal factors of transcranial stimulation. Each animal was given 24 hours to recover.

The following day, each animal was individually anesthetized with isoflurane (1\%). The anodal electrode was then connected to a commercial neural stimulator (Multi Channel System STG 1008). A reference surface electrode $(2.5 \mathrm{~cm} 2)$ was constructed from a flexible $2 \times 3.5$ " self-adhering electrode sheet designed for transcutaneous electrical nerve stimulation from Medical Products, Inc. Each electrode was attached to the rat pup's chest after the hair had been removed using a small Velcro fixed vest. The HI pups were then separated into two groups: nonstimulated (HI) and tDCS stimulated (HI-tDCS). For the tDCS treated group, the stimulating parameters were the same for every animal, with a specific current protocol of short anodal interstimulation intervals. Safety limits of tDCS in rats have suggested current levels between $143 \mu \mathrm{A}$ and $286 \mu \mathrm{A}$ with stimulation times ranging from 10 to 90 minutes that do not produce tissue damage even after daily use [29]. An electric current followed a 13-3-13 configuration in which current slowly ramped up over 3 minutes to a total of $200 \mu \mathrm{A}$, maintained for 13 minutes, turned off for 3 minutes, then immediately back on at $200 \mu \mathrm{A}$ for another $13 \mathrm{~min}$ before it was ramped down to $0 \mu \mathrm{A}$ (Figure $5 \mathrm{~b}$ ). Previous studies have shown that repetitive interval currents like that used here extend beneficial effects after the stimulation [30]. Each animal was stimulated once a day for a total of one week. The non-stimulated HI and Sham groups underwent anesthesia, were connected to the stimulator for the same duration, but without current being applied. After a short recovery period following anesthesia, all animals were returned to the dams.

\section{Neurobehavioral assessments}

To determine if tDCS stimulation produced positive changes in motor performance for the hypoxic-ischemic rat pups, we compared a number of neurological signs, reflexes, and motor coordination measures between the age-matched sham pups, age-matched nontDCS treated pups, and age-matched tDCS treated pups were performed using the following common neurobehavioral tests for neonatal hypoxic-ischemic rats. The pups underwent evaluation testing using the negative geotaxis test, a catwalk gait analysis, and grip strength testing, thus primarily examining motor function. Negative geotaxis was performed for each rat approximately 15 minutes before anesthesia.

Weight: Total body weight was recorded daily (Veritas - S Series Precision Balances) beginning from the day of postnatal HI surgery to the end of the stimulation period. Each animal's weight was used to ensure the animal's health and as an indirect measure to determine whether any improvement in motor performance from tDCS treatment resulted in increased physical function that included appetitive behaviors in the home cage.

Negative Geotaxis: Negative Geotaxis test is a reliable tool for detecting motor capabilities of these animals [31]. A negative Geotaxis
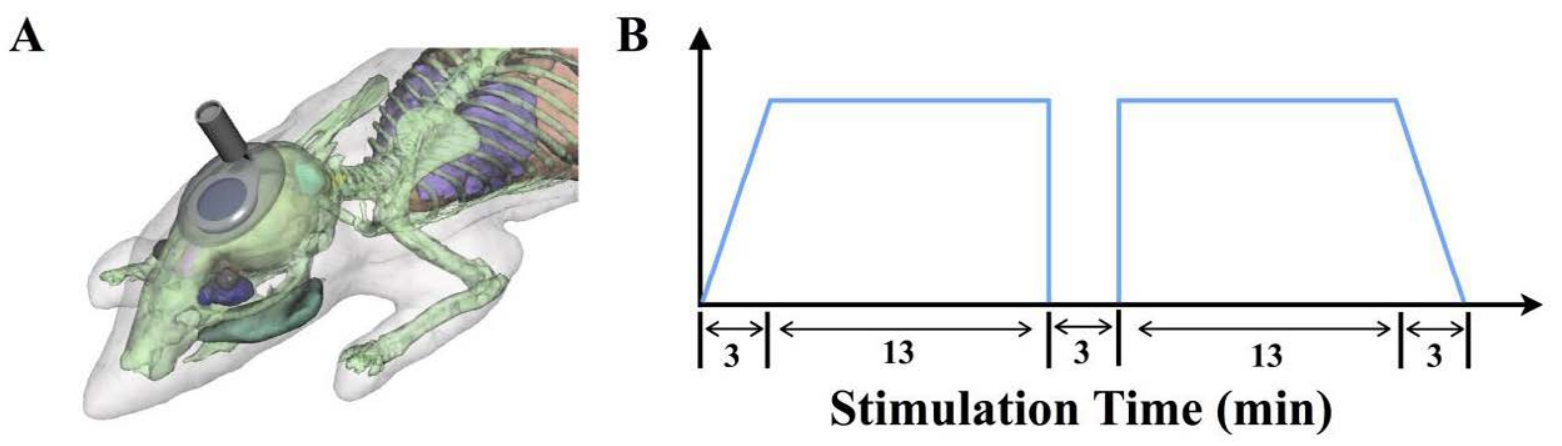

Figure 5. (a) Illustration of ring-electrode and placement over the skull in rat pups during tDCS treatment following HI injury. A small ring electrode was placed under the scalp and cemented into place over the skull and corresponding ischemic injury site. This arrangement maintained the position and corresponding stimulation site over the course of the stimulations. (b) Electrical Stimulation Parameters: Electronic current was ramped over 3 minutes to a total of $200 \mu \mathrm{A}$. Current remained at $200 \mu \mathrm{A}$ for 13 minutes, off for 3 minutes, then immediately back to $200 \mu \mathrm{A}$ for another 13 before ramping down to $0 \mu \mathrm{A}$. 
test [32] was performed each day from P19 to the end of the stimulation week at P29. The handler placed each pup's nose facing down on the center a fine-grit board set at a 45-degree angle and then released. A digital stopwatch was used to record the time required for the pup to pivot and completely turn its body upwards. The test was performed five times for each animal and average recorded each day.

Grip Strength Test: The Grip Strength test is a common metric used to quantify the effect of HI on a pup's muscular system and measure used in the pediatric evaluation of human infants of stroke and cerebral palsy [33]. First, the paw on the same side of the injury (left) was taped down to the body of the animal and paw on the contralateral side tested. Using a grip strength-testing device (Bioseb In Vivo Instruments, Model BIO-GS3) the handler briefly allows for the pup to make contact with a spatially designed metal grid and then pulls them off as they begin to gain grip. Highly accurate sensors sample at a rate of $100 \mathrm{~Hz}$ to determine the maximal peak force developed by the animal's grip of the grid as it is being removed. Each pup was tested on the device 5 times and the results are averaged on three separate days: Once before stimulation (P21), in the middle of the stimulation week (P25), and after stimulation (P29).

Catwalk Gait Analysis (CGA): The Catwalk procedure is an automated gait analysis method that allows easy quantitation of a large number of locomotor parameters during normal walking behavior. The Catwalk procedure was initially developed by Hamers, F.P., et al., in 2001[34] to investigate locomotor function following spinal cord injury in rats. The Catwalk is a commercially available hardware and software system (Noldus Information Technology, CatWalk XT, Leesburg, VA). In this experimental procedure rats are allowed to walk along a 1.25-meter enclosed walkway with a glass plate as the walking surface. This glass plate emits light from a light emitting diodes, such that light is internally reflected except at those areas where the animal makes contact with the glass plate. A high-speed color camera that is positioned underneath the glass plate captures temporal and spatial data on the relative brightness of contact and non-contact areas. The Noldus software records and translates the digital values into indices of motor function.

The goal was to have the rat walk from one end of the runway to the other end in less than 10- seconds without stopping. A minimum of three footstep cycles, where one complete cycle is defined as placement of each of 4 paws, was collected during this 10 -second time period. Three 10-second trials of a complete footstep cycles were needed for a complete analysis. The total time required per testing session did not exceed 5-minutes. All three groups of animals were tested once before stimulation (P21) and once after the 7 days of stimulation were complete in a subset of animals ( $\operatorname{sham~} n=2, \mathrm{HI} n=2$, and HI-tDCS $n=2$ ). The Catwalk measures examined in this study were:

Base of Support: average width $(\mathrm{cm})$ between the Front Paws of each animal when walking and is typically smaller in $\mathrm{HI}$ animals.

Print Area: calculated surface area of each Hind Paw when the animal is making contact while walking and is often larger in animals with HI deficit.

Phase Dispersion Area: which is the mean temporal placement of two paws within two consecutive initial contacts, used to measure the coordination of the animal's paw placement and is generally much higher in HI animals.

\section{Brain derived neurotropic factor}

The expression of brain-derived neurotropic factor (BDNF) is commonly used as an indirect measure of neuronal plasticity in the brain. There are now a number of reports supporting tDCS's effect may be due to plasticity as measured through changes in BDNF expression $[5,19]$. Brain-derived neurotropic factor was measured using the Promega BDNF Emax Elisa kit (Thermo Scientific, Modified Lowry Protein Assay). Protein samples were collected and diluted using the brains from HI injury animals and HI-tDCS treated animals using a subset of animals. We collected the right and left cortex and the right and left hippocampus in cold SEB buffer: $250 \mathrm{mM}$ Sucrose, $10 \mathrm{mM}$ Hepes $\mathrm{KoH}$ (pH 7.5), 1mM EGTA with protease inhibitors added. The tissue was then homogenized and sonicated for 30 seconds, centrifuged at $1000 \mathrm{~g}$ for 5 minutes, and supernatant stored at $-80^{\circ}$ Celsius. The concentration of BDNF was determined using a standard Modified Lowry Protein Assay protocol (Termo Scientific). BDNF is estimated in picograms of BDNF per microgram of protein and BDNF ratio is calculated as the ratio of BDNF in injured vs uninjured cortex. Samples were collected at two time points, $\mathrm{P} 30$ ( $\mathrm{n}=5$ for HI-tDCS, $\mathrm{n}=4$ for HI) and P36 ( $\mathrm{n}=2$ for HI-tDCS, $\mathrm{n}=1$ for HI).

\section{Statistics}

A normal theory linear mixed model framework was used, which is a two-way ANOVA with subject-specific random effects, with variables of group and time point was used for the Catwalk analysis. The full statistical model: $Y i j k=\alpha i+\beta j+\gamma k+(\beta \gamma) j k+\epsilon i j k$, where $Y i j k$ is the outcome variable of interest, $\alpha i$ is the subject-specific random intercept, $\beta j$ is the main effect of treatment group, $\gamma k$ is the effect of the time point, and $(\beta \gamma) j k$ is the interaction term. Statistical differences between were considered significant at $\mathrm{p}<0.05$.

\section{Statement of financial support}

This study was supported by grants from the National Institutes of Health NINDS R01NS077004-01.

\section{Disclosure statement}

There are no financial ties to products in the study or any potential/ perceived conflicts of interest from any of the authors.

\section{Category of study}

Translational Research.

\section{References}

1. Lynch JK,Hirtz DG, DeVeber G, Nelson KB (2002) Report of the National Institute of Neurological Disorders and Stroke workshop on perinatal and childhood stroke. Pediatrics 109: 116-123.[Crossref]

2. Veerbeek JM, van Wegen E, van Peppen R, van der Wees PJ, Hendriks E, et al. (2014) What is the evidence for physical therapy poststroke? A systematic review and metaanalysis. PLoS One 9: e87987.[Crossref]

3. Johnson MD, Lim HH, Netoff TI, Connolly AT, Johnson N, et al. (2013) Neuromodulation for brain disorders: challenges and opportunities. IEEE Trans Biomed Eng 60: 610-624.[Crossref]

4. Brem AK, Fried PJ, Horvath JC, Robertson EM, Pascual-Leone A (2014) Is neuroenhancement by noninvasive brain stimulation a net zero-sum proposition? NeuroImage 85: 1058-1068.[Crossref]

5. Brunoni AR, Nitsche MA, Bolognini N, Bikson M, Wagner T, et al. (2012) Clinical Research with Transcranial Direct Current Stimulation (tDCS): Challenges and Future Directions. Brain Stimul 5: 175-195. [Crossref]

6. Hasan A, Falkai P, Wobrock T (2013) Transcranial brain stimulation in schizophrenia: 
targeting cortical excitability, connectivity and plasticity. Curr Med Chem 20: 405-413. [Crossref]

7. Reis J, Robertson EM, Krakauer JW, Rothwell J, Marshall L, et al. (2008) Consensus: can transcranial direct current stimulation and transcranial magnetic stimulation enhance motor learning and memory formation? Brain Stimul 1: 363-369.[Crossref]

8. Thickbroom GW, Mastaglia FL (2009) Plasticity in neurological disorders and challenges for noninvasive brain stimulation (NBS). J Neuroeng Rehabil 6: 4. [Crossref]

9. Flöel A (2014) tDCS-enhanced motor and cognitive function in neurological diseases. Neuroimage 85 Pt 3: 934-947.[Crossref]

10. Nitsche MA, Cohen LG, Wassermann EM, Priori A, Lang N, et al. (2008) Transcranial direct current stimulation: State of the art 2008. Brain Stimul 1: 206-223.[Crossref]

11. Schlaug G, Renga V, Nair D (2008) Transcranial direct current stimulation in stroke recovery. Arch Neurol 65: 1571-1576.[Crossref]

12. Marquez J, Van Vliet P, McElduff P, Lagopoulos J, Parsons M (2015) Transcranial direct current stimulation (tDCS): does it have merit in stroke rehabilitation? A systematic review. International Journal of Stroke 10: 306-316.

13. Nitsche MA, Nitsche MS, Klein CC, Tergau F, Rothwell JC, et al. (2003) Level of action of cathodal DC polarisation induced inhibition of the human motor cortex. Clin Neurophysiol 114: 600-604.[Crossref]

14. Krishnan C, Santos L, Peterson MD, Ehinger M (2015) Safety of noninvasive brain stimulation in children and adolescents. Brain Stimul 8: 76-87.[Crossref]

15. Wittenberg GF (2009) Neural plasticity and treatment across the lifespan for motor deficits in cerebral palsy. Dev Med Child Neurol 51: 130-133. [Crossref]

16. Johnston MV, Ferriero DM, Vannucci SJ, Hagberg H (2005) Models of cerebral palsy: which ones are best? J Child Neurol 20: 984-987.[Crossref]

17. Nitsche MA, Paulus W (2001) Sustained excitability elevations induced by transcranial DC motor cortex stimulation in humans. Neurology 57: 1899-1901.[Crossref]

18. Stagg CJ, Best JG, Stephenson MC, O'Shea J, Wylezinska M, et al. (2009) Polaritysensitive modulation of cortical neurotransmitters by transcranial stimulation. $J$ Neurosci 29: 5202-5206. [Crossref]

19. Fritsch B, Reis J, Martinowich K, Schambra H, Ji Y, et al. (2010) Direct current stimulation promotes BDNF-dependent synaptic plasticity: potential implications for motor learning. Neuron 66(2): 198-204.[Crossref]

20. Giordano J, Bikson M, Kappenman ES, Clark VP, Coslett HB, et al. (2017) Mechanisms and Effects of Transcranial Direct Current Stimulation. Dose Response 15: 1559325816685467.[Crossref]
21. Chen CF, Bikson M, Chou LW, Shan C, Khadka N, et al. (2017) Higher-order power harmonics of pulsed electrical stimulation modulates corticospinal contribution of peripheral nerve stimulation. Sci Rep 7: 43619.[Crossref]

22. Miranda PC, Lomarev M, Hallett M (2006) Modeling the current distribution during transcranial direct current stimulation. ClinNeurophysiol117: 1623-1629. [Crossref]

23. Underwood, Emily (2016) Cadaver study casts doubts on how zapping brain may boost mood, relieve pain. Science, [online] April 20.

24. Bikson M, Truong DQ, Mourdoukoutas AP, Aboseria M, Khadka N, et al. (2015) Modeling sequence and quasi-uniform assumption in computational neurostimulation. Progress in brain research 222: 1-23.

25. Rice JE 3rd, Vannucci RC, Brierley JB (1981) The influence of immaturity on hypoxicischemic brain damage in the rat. Ann Neurol 9: 131-141.[Crossref]

26. Vannucci RC, Vannucci SJ (2005) Perinatal hypoxic-ischemic brain damage: evolution of an animal model. Dev Neurosci 27: 81-86. [Crossref]

27. Taniguchi H, Andreasson K (2008) The hypoxic ischemic encephalopathy model of perinatal ischemia. J Vis Exp 19: e955-e955. [Crossref]

28. Datta A, Bansal V, Diaz J, Patel J, Reato D, et al. (2009) Gyri-precise head model of transcranial DC stimulation: Improved spatial focality using a ring electrode versus conventional rectangular pad. Brain Stimul 2: 201-207. [Crossref]

29. Liebetanz D, Koch R, Mayenfels S, Konig F, Paulus W, et al. (2009) Safety limits of cathodal transcranial direct current stimulation in rats. ClinNeurophysiol 120: 11611167. [Crossref]

30. Monta-silva K, Kuo MF, Liebetanz D, Paulus W, Nitsche MA (2010) Shaping the optimal repetition interval for cathodal transcranial direct current stimulation (tDCS). $J$ Neurophysiol103: 1735-1740.[Crossref]

31. Lubics A, Reglodi D, Tamás A, Kiss P, Szalai M, et al. (2005) Neurological reflexes and early motor behavior in rats subjected to neonatal hypoxic-ischemic injury. Behav Brain Res 157: 157-165. [Crossref]

32. Iwai M, Chen J (2012) Neurobehavioral Assessments of Neonatal HypoxiaIschemia. Animal Models of Acute Neurological Injuries II: Injury and Mechanistic Assessments 2: 223-228.

33. Häger-Ross C, Rösblad B (2002) Norms for grip strength in children aged 4-16 years. Acta Paediatr 91: 617-625.[Crossref]

34. Hamers FP, Lankhorst AJ, van Laar TJ, Veldhuis WB, Gispen WH (2001) Automated quantitative gait analysis during overground locomotion in the rat: its application to spinal cord contusion and transection injuries. J Neurotrauma 18: 187-201. [Crossref]

Copyright: (C2017 Anderson CL. This is an open-access article distributed under the terms of the Creative Commons Attribution License, which permits unrestricted use, distribution, and reproduction in any medium, provided the original author and source are credited. 